\title{
An Ecological Analysis of the Impact of Temperature Inversion on Emergency Department Visits for Respiratory Syndromes and Subsyndromes using BioSense 2.0 Frontend Data
}

\author{
Anne Burke*, David Jackson and Allyn K. Nakashima \\ Bureau of Epidemiology, Utah Department of Health, Salt Lake City, UT, USA
}

\section{Objective}

To determine the association between emergency department (ED) visits for the respiratory syndrome and subsyndromes and air quality indices (AQI) for fine particle pollution in Salt Lake County, UT using frontend BioSense 2.0 data.

\section{Introduction}

During the winter months, Utah experiences a temperature inversion which traps pollutants, such as fine particle pollution (PM ${ }_{25}$ ), in the Salt Lake Valley. A previous study determined the impact of inversion on ED visits for asthma, ${ }^{1}$ however similar phenomena have yet to be examined using the BioSense 2.0 syndromic surveillance system. While similar studies utilize a time-stratified case-crossover design, the absence of individually identifiable information on the dashboard precludes the utilization of this methodology. Using BioSense 2.0 frontend data, an ecological study design may allow for analyses to determine the impact of inversion on ED visits for respiratory syndrome and subsyndromes from submitting facilities in Salt Lake County, UT.

\section{Methods}

Numbers and proportions of visits were tabulated for the BioSense 2.0 respiratory syndrome and subsyndromes (asthma, bronchitis and bronchiolitis, chest pain, cough, cyanosis and hypoxemia, dyspnea, hemoptysis, influenza-like illness (ILI), otitis media, pleurisy, pneumonia and lung abscess, respiratory failure, respiratory syncytial virus (RSV), and upper respiratory infections) for January 2012 to February 2014. Particulate concentration data for $\mathrm{PM}_{2.5}$ in Salt Lake County, UT were be obtained from the EPA website and converted to AQI for the same dates. Voluntary and mandatory action days were identified by $\mathrm{PM}_{2.5}(15-25$ and $>25 \mu \mathrm{g} / \mathrm{m} 3$, respectively) and analysis of variance (ANOVA) models were performed to determine if the proportion of ED visits differed significantly by action day status. The CDC's C2 aberration detection methodology was used to determine dates when the number of ED visits for respiratory events exceeded the alarm threshold. These days were examined in relation to AQI using ANOVA models in order to determine if there was a significant association between days exceeding the threshold and air quality. A linear regression analysis was conducted to determine the association between BioSense 2.0 respiratory syndrome and subsyndromes and AQI when controlling for seasonality.

\section{Results}

A total of 80 voluntary and 88 mandatory action days occurred during this time period. The mean proportion of total visits for all respiratory syndromes and subsyndromes except for respiratory failure and otitis media increased significantly with more severe action day status $(\mathrm{p}<0.05)$. Overall, 85 alarms were triggered for respiratory syndromes and subsyndromes during this time period. The mean AQI was significantly greater on days in which the proportion of ED visits exceeded the alarm threshold for ILI, pleurisy, and respiratory failure $(\mathrm{p}<0.05)$. After adjusting for seasonality, the AQI was significantly associated with the proportion of ED visits for RSV, ILI, and the respiratory syndrome $(\mathrm{p}<0.05)$.

\section{Conclusions}

Regardless of analytical technique, the proportion of ED visits for the BioSense 2.0 respiratory syndrome and ILI were significantly associated with AQI; however, the association of other subsyndomes differed by method of analysis and the inclusion of control variables. These data suggest that voluntary and mandatory action days were associated with higher proportions of respiratory visits for most respiratory conditions. Alarm events, on the other hand, were most likely to be associated with AQI for ILI, pleurisy, and respiratory failure; this data may be useful to ED practices in Salt Lake County. RSV and ILI remained significantly associated with AQI when controlling for seasonality. The association between these infectious conditions and AQI may inform the use of syndromic data to conduct disease surveillance during the influenza and RSV seasons. Overall, these data suggest that frontend BioSense 2.0 data may serve useful to ecological analyses for air quality issues, such inversions in Salt Lake County, UT. Because of its scope and differing characteristics of respiratory subsyndromes, breaking the respiratory syndrome down into its various subsyndromes for analysis may provide more a more accurate representation of its association with air quality.

\section{Keywords}

BioSense 2.0; Respiratory syndrome; Air pollution

\section{References}

1. Beard JD, Beck C, Graham R, Packham SC, Traphagan M, Giles RT, Morgan JG. Winter temperature inversions and emergency department visits for asthma in Salt Lake County, UT, 2003-2008. Environmental Health Perspectives 2012;120(10):1385-1390.

\footnotetext{
*Anne Burke

E-mail: aburke@utah.gov
} 